Shuwen Zhou $ه$

Siqi Zhang

Qingming Chen

https://doi.org/10.21278/TOF.43Si101

ISSN 1333-1124

eISSN 1849-1391

\title{
VEHICLE ABS EQUIPPED WITH AN EMB SYSTEM BASED ON THE SLIP RATIO CONTROL
}

\begin{abstract}
Summary
The brake system plays a key role in the safe operation of an automobile. As an important development direction of brake systems, electromechanical braking devices have attracted great interest because of their many advantages. Braking dynamics based on a twowheel vehicle model are analyzed in this paper. Then, an electromechanical brake device is designed and a simulation model of a vehicle ABS based on the electromechanical brake device is established. A sliding mode control system is designed to regulate the anti-lock braking system. The control algorithm is verified using a variety of operating conditions in vehicle dynamics simulation software. The simulation results show that the control method proposed in this paper can improve the braking effect.
\end{abstract}

Key words: $\quad$ Electromechanical brake; Sliding mode control; PID; Anti-lock braking system; Slip ratio

\section{Introduction}

An anti-lock braking system (ABS) allows a vehicle to stop quickly under slippery road conditions and is a key technology to improve vehicle braking safety. Research on the ABS control algorithm is an important for improving vehicle braking performance. Currently, the major parameters of the ABS control algorithm include logic threshold control, classic PID control, fuzzy control, sliding mode variable structure control, neural network control, and others. However, due to the limitations of the execution speed of the hydraulic actuator and transmission delay of the hydraulic braking lines for conventional hydraulic braking systems, most practical products still use logic threshold control based on experience. However, the logic threshold control algorithm requires many road tests and cannot make full use of the road adhesion capability.

An electromechanical braking (EMB) system uses electricity as the energy source, which transmits energy by an electric wire while the data line transmits a signal. The use of electricity removes the complicated hydraulic pipeline and hydraulic components from the system. Therefore, electronic mechanical brake systems are more suitable for electric 
vehicles. An electromechanical braking system, which is the future developmental direction of braking systems, is a great choice for electric vehicles and is gradually becoming a research focus in the automotive industry.

Major automotive companies and research institutes have developed various EMB system actuator prototypes and have conducted road tests by applying the systems in vehicles. Our laboratory has also developed an electromechanical braking device and applied for a patent for its design.

The EMB system actuator utilizes the motor as its power drive mechanism, which is particularly useful for the control algorithm based on slip ratio control and can give full control to ABS because of its fast response speed. In recent years, variable structure control has been successfully applied in the simulation of various types of mechanical and electrical products. Some types of sliding mode controllers have also been designed for automobile ABS $[1,2]$. In traditional automotive ABS control systems, there are many sliding mode controllers based on equivalent torque that use the saturation function and other methods to eliminate noise due to variable structure control [3-5]. For hybrid and electric cars, simulation studies still focus on hydraulic (pneumatic) braking systems; few sliding mode controllers have been designed for hybrid or electric vehicles equipped with EMB systems [6-8]. Among them, study focused on a quarter vehicle model based on a fuzzy control method and the sliding mode control (SMC) method [9-11].

Based on the classical mechanics of a two-wheel vehicle model, a model was built to analyze vehicle brake maneuvers, which includes the effect of longitudinal load transfer during braking. A simulation model of an electromechanical brake device was built in vehicle dynamics simulation software, and the control model of the anti-lock brake system was designed in Simulink. Co-simulation was carried out based on the simulation model and control model. Then, a sliding mode control algorithm was designed based on the equivalent torque. By comparing the simulation model with the classical PID control algorithm, it was demonstrated that the electromechanical braking device had an incomparable advantage over the traditional hydraulic braking system. At the same time, the comparison verified the algorithm based on slip ratio control, including sliding mode control and classic PID control. It was concluded that the sliding mode control as better than the other algorithms for vehicles equipped with EMB systems.

\section{EMB dynamics model}

The EMB system mainly consists of four of the same EMB actuators installed on all four wheels of the vehicle. Considering the impact of axial load transfer during braking, this paper first designs controllers for the front and rear wheels on one side of the vehicle. Then, slightly modified controllers are applied to wheels on the other side of the vehicle.

\subsection{Two-wheel vehicle model}

Vehicle and wheel braking dynamics analyses are conducted using classical mechanics, as shown in Figure 1. The moment of inertia generated by the rotating mass during deceleration is ignored. According to D'Alembert's principle, vehicle dynamic equations can be established according to the longitudinal direction of travel and the rotation directions of the two wheel spindles [12]. 
The vehicle dynamic equation is,

$$
m \dot{v}=-F_{f f}-F_{f r}-F_{a}
$$

The wheel braking dynamics equation is,

$$
\begin{gathered}
J_{\omega f} \dot{\omega}_{f}=R F_{f f}-T_{b f}-T_{r f} \\
J_{\omega r} \dot{\omega}_{r}=R F_{f r}-T_{b r}-T_{r r}
\end{gathered}
$$

where $m$ - half vehicle mass, $v$ - vehicle speed, and $F_{f f}, F_{f r}$ - longitudinal friction of the front and rear wheels, respectively. $F_{a}$ - aerodynamic resistance, $J_{\omega f}, J_{\omega r}$ - moment of inertia of the front and rear wheels, respectively. $\omega_{f}, \omega_{r}$ - angular velocity of the front and rear wheels, respectively, and $T_{b f}, T_{b f}$ - brake torque of the front and rear wheels, respectively. $T_{r f}, T_{r r}$ - rolling resistance torque on the front and rear wheels, respectively.

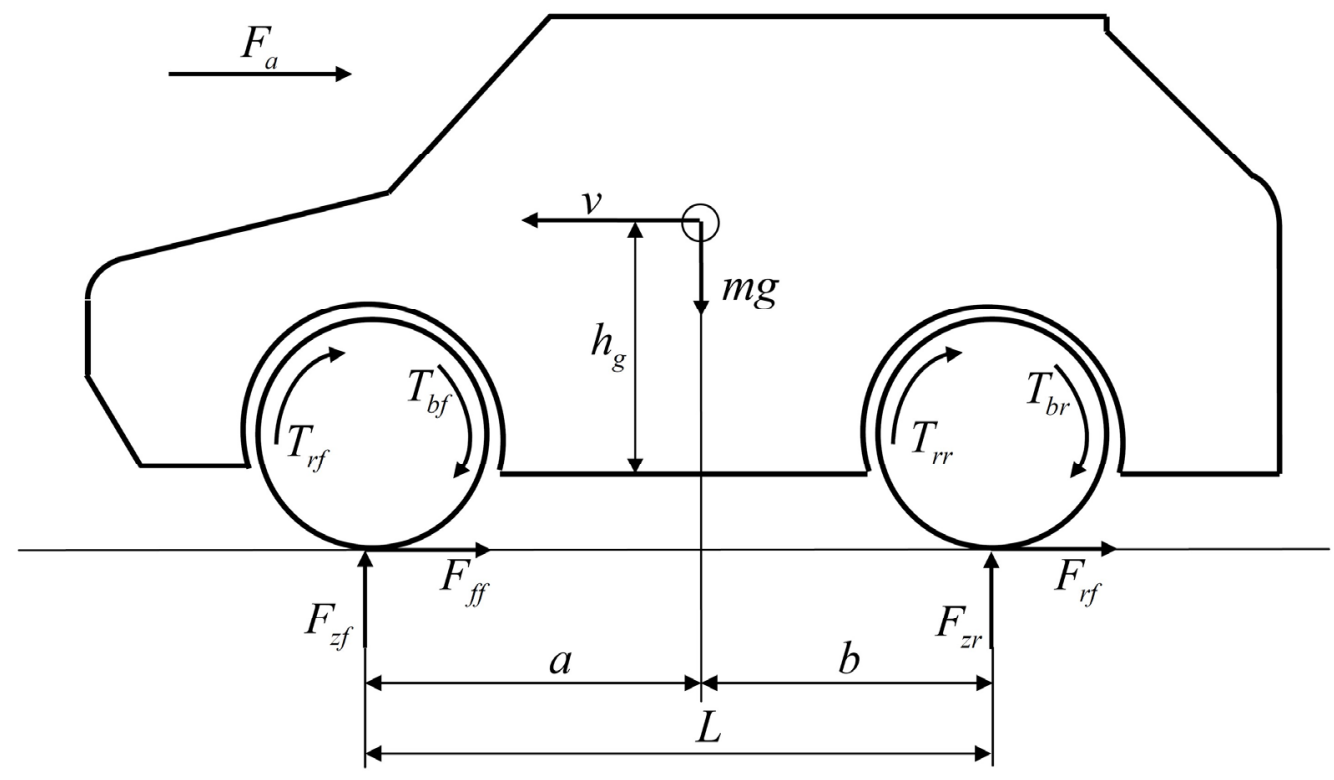

Fig. 1 Two-wheel vehicle model

The aerodynamic resistance [13] is,

$$
F_{a}=\frac{1}{2} C_{D} A_{f} \rho_{a} v^{2}
$$

where, $C_{D}$ - air resistance coefficient, $A_{f}$ - vehicle frontal area, and $\rho_{a}$ - air density.

The longitudinal friction of the front and rear wheels is,

$$
\begin{aligned}
& F_{f f}=\mu F_{z f} \\
& F_{f r}=\mu F_{z r}
\end{aligned}
$$

where, $\mu$ - braking effort coefficient of road adhesion and $F_{z f}, F_{z f}$ - normal reaction on the front and rear wheels from the road. 
During braking, when considering the load transfer from the rear wheel to the front wheel, the normal loads on the front and rear wheels can be expressed as,

$$
\begin{aligned}
& F_{z f}=\frac{1}{L}\left[m g b+h_{g}\left(m \dot{v}-F_{a}\right)\right] \\
& F_{z r}=\frac{1}{L}\left[m g a-h_{g}\left(m \dot{v}-F_{a}\right)\right]
\end{aligned}
$$

where $L$ - wheelbase; $g$ - acceleration of gravity; $a, b$ - distance between the front axle and rear axle to the center of mass, respectively; and $h_{g}$ - height of the center of mass from the ground.

The slip rate is not only the proportion of the sliding component in the wheel rotation but is also an important indicator that reflects the level of wheel lock. The longitudinal slip rate of the wheel is,

$$
\lambda_{i}=\frac{v-\omega_{i} R}{v} \times 100 \%
$$

where $i=f, i=r$ - representation of the variables of the front and rear wheels.

\subsection{Tire model}

Tires are the only components that make contact with the vehicle and road surface; in addition to air resistance, relatively all of the other external forces are generated by the interaction of the tire and road surface. Therefore, the mechanical properties of the tire have a vital influence on the braking performance of the car.

To simplify the calculations and analysis, the empirical tire model proposed by Pacejka et al. was used in this paper [14-18]. The longitudinal adhesion coefficient in this tire model is

$$
\mu\left(\lambda_{i}\right)=f+D \sin \left\{C \arctan \left[B \lambda_{i}-E\left(B \lambda_{i}-\arctan B \lambda_{i}\right)\right]\right\}
$$

where $f$ - coefficient of static friction, $D$ - peak factor, $C$ - curve shape factor, $B$ - stiffness factor, and $E$ - curve curvature factor.

Generally, $f$ is equivalent to a pure rolling wheel adhesion coefficient and is always taken as $0 . D, C, B$, and $E$ are constants related to the road surface, which are determined from road experiments.

\subsection{Electromechanical brake device model}

This paper employs an electromechanical braking device developed in our laboratory [19]. The actuator prototype is shown in Figure 2 and Figure 3. The modular structure method was used to design the actuator, which includes the power driving mechanism, reduction mechanism and motion conversion mechanism.

At the beginning of the braking phase, the control unit will determine the size and direction of the control current by analyzing the sensor signals. The motor will generate the appropriate braking torque, which is needed by each wheel in this state according to the 
current. Then, through the reduction mechanism and motion conversion mechanism, the final braking torque will be passed to the braking disc. In the brake release phase, the control current is reversed; the motor is put in reverse, the ball screw returns to its original position and the clearance is self-adjusted. Therefore, the EMB system actuator model can be simplified as a first-order system and a delay function. The transfer function [20] is

$$
G(s)=\frac{T_{b}}{I}=e^{-\tau s} \frac{k}{T s+1}
$$

where $T_{b}$ - output torque of the electromechanical brake device, $I$ - control current, $\tau$ mechanical transmission delay constant, $k$ - amplification factor of the electromechanical brake device, and $T$ - motor drive model time constant.

Based on the motor drive and mechanical drive models, the amplification factor of the electromechanical brake device shown in Figure 2 can be obtained [21, 22] as

$$
k=\frac{T_{b}}{I_{k}}=9.55 \cdot 4 \pi \cdot K_{e} \cdot i \cdot \eta_{x} \cdot \eta_{s} \cdot k_{p} / p_{h}
$$

where $I_{k}$ - stall current of the drive motor, $K_{e}$ - coefficient of the counter electromotive force, $i$ - two-stage gear reduction mechanism transmission ratio, $\eta_{x}$ - mechanical efficiency of the reduction mechanism, $\eta_{s}$ - drive efficiency of the ball screw, $k_{p}$ - braking factor, and $p_{h}$ - lead of the ball screw.

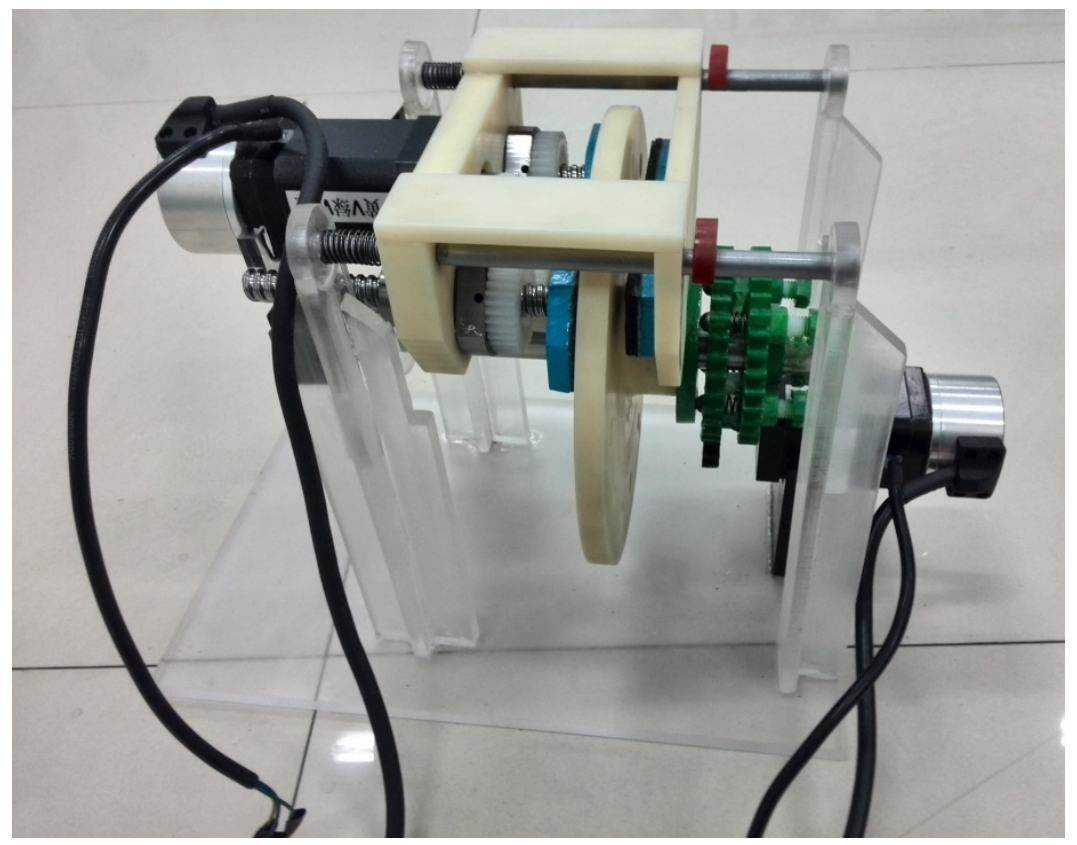

Fig. 2 Electro-mechanical brake device 
a)

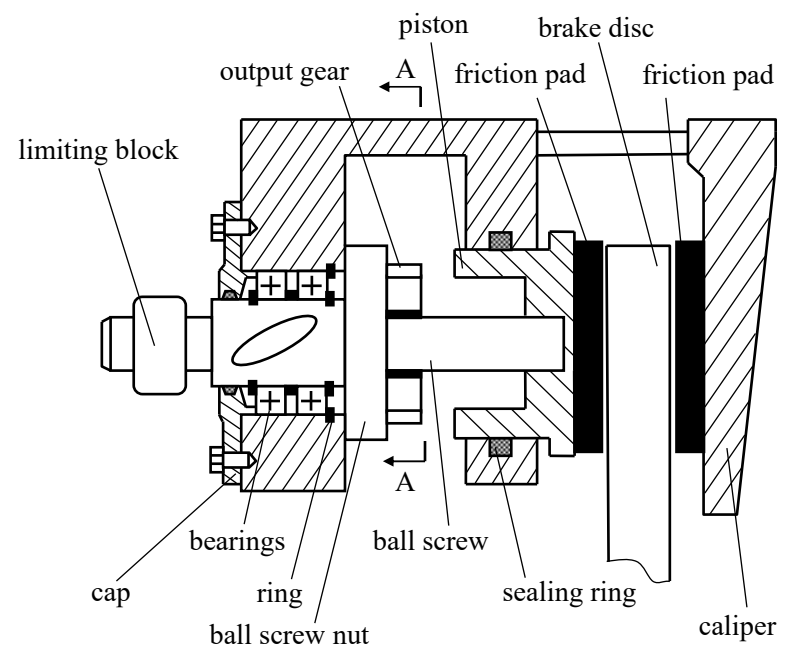

b)

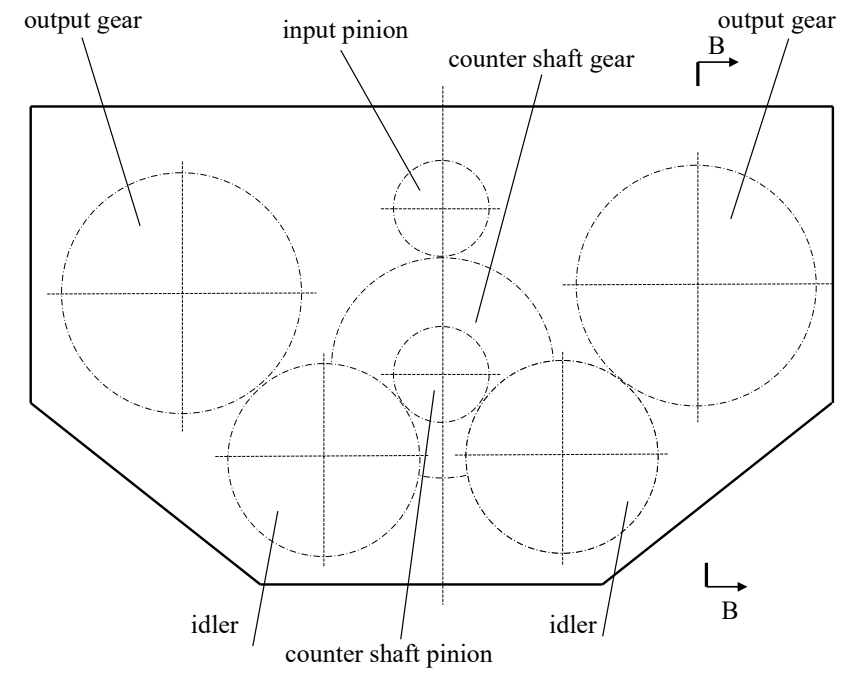

Fig. 3 Electro-mechanical brake schematic diagram

\section{Sliding mode controller design}

The task of ABS is to control the actual slip ratio of the wheel in the vicinity of the ideal slip ratio and to obtain the maximum longitudinal adhesion coefficient and high lateral adhesion coefficient. In this case, ABS can not only prevent the wheel from locking and shorten the brake distance but also obtain a larger lateral adhesion coefficient, which improves the handling and stability of the vehicle during emergency braking.

Sliding mode control is a special type of nonlinear control and can be purposefully changed according to the current state of the system, forcing the system into a predetermined trajectory "sliding mode" state. Therefore, to ensure the existence of the sliding mode, we first need to design a switching function $s$ in the sliding mode controller design.

In this paper, it is assumed that the vehicle brakes during an emergency at a certain initial velocity on dry asphalt, the corresponding desired slip rate of which is approximately 0.2. Therefore, the desired slip rate $\lambda_{d}$ is set as 0.2 in this paper. The target of the control system is to eliminate the tracking error and ensure that the slip rate remains near the desired rate. Therefore, the switching function chosen in this paper is

$$
s_{i}=\lambda_{i}-\lambda_{d}
$$


The derivation of equation (9) can be obtained as

$$
\dot{\lambda}_{i}=\frac{1}{v}\left[\left(1-\lambda_{i}\right) \dot{v}-\dot{\omega}_{i} R\right]
$$

Equation (1) - (3) can be rewritten as

$$
\begin{aligned}
& \dot{v}=-\frac{F_{a}}{m}-\sum_{i=f, r} \frac{F_{f i}}{m} \\
& \dot{\omega}_{i}=-\frac{T_{r i}}{J_{\omega i}}+\frac{R}{J_{\omega i}} F_{f i}-\frac{1}{J_{\omega i}} T_{b i}
\end{aligned}
$$

Substitute equation (5) and (6) into equation (15) and (16)

$$
\begin{aligned}
& \dot{v}=-f_{1}-\sum_{i=f, r} f_{2 i} \mu\left(\lambda_{i}\right) \\
& \dot{\omega}_{i}=-f_{3 i}+f_{4 i} \mu\left(\lambda_{i}\right)-a_{1 i} T_{b i}
\end{aligned}
$$

where $f_{1}=\frac{F_{a}}{m}, f_{2 i}=\frac{F_{z i}}{m}, f_{3 i}=\frac{T_{f i}}{J_{\omega i}}, f_{4 i}=\frac{F_{z i}}{J_{\omega i}} R, a_{1 i}=\frac{1}{J_{\omega i}}$.

We substitute equation (17) and (18) into equation (14)

$$
\dot{\lambda}_{i}=f_{5 i}-f_{6 i} \mu\left(\lambda_{f}\right)-f_{7 i} \mu\left(\lambda_{r}\right)+f_{8 i} T_{b i}
$$

where $f_{5 i}=\frac{f_{3 i} R-\left(1-\lambda_{i}\right) f_{1}}{v}, f_{6 i}=\frac{f_{4 i} R+\left(1-\lambda_{i}\right) f_{2 i}}{v}, f_{7 i}=\frac{\left(1-\lambda_{i}\right) f_{2 i}}{v}, f_{8 i}=\frac{a_{1 i} R}{v}$.

The equivalent braking torque of the sliding mode control can be derived from equation (13), equation (19) and $\dot{s}_{i}=0$

$$
T_{\text {beqi }}=\frac{f_{6 i} \mu\left(\lambda_{f}\right)+f_{7 i} \mu\left(\lambda_{r}\right)-f_{5 i}}{f_{8 i}}
$$

The system moving point will move in the designed switching surface under the action of the equivalent moment. However, when the system deviates from the switching surface, it is necessary to design a control law to reliably introduce the initial state of the system point into the sliding mode and keep it moving within it, so that the system satisfies the reaching condition of sliding mode control. The braking torque can be defined as

$$
T_{b i}=T_{b e q i}-K \operatorname{sgn}(s)
$$

To meet the arrival condition of the sliding mode control, a Lyapunov stability condition is adopted as

$$
s \dot{s} \leq 0
$$

Substituting equation (19) into equation (22)

$$
s\left(f_{5 i}-f_{6 i} \mu\left(\lambda_{f}\right)-f_{7 i} \mu\left(\lambda_{r}\right)+f_{8 i} T_{b i}\right) \leq 0
$$


Substituting equation (21) into equation (23)

$$
s\left[f_{5 i}-f_{6 i} \mu\left(\lambda_{f}\right)-f_{7 i} \mu\left(\lambda_{r}\right)+f_{8 i}\left(T_{\text {beqi }}-K \operatorname{sgn}(s)\right)\right] \leq 0
$$

Substituting equation (20) into equation (24)

$$
-f_{8 i} K|S| \leq 0
$$

where $f_{8 i}=\frac{R}{J_{\omega i} v}$ is clearly greater than 0 . To meet the arrival condition of sliding mode control, $K$ is taken as $\frac{v_{0} J_{\omega i \max }}{R} \eta$ (where, $v_{0}$ - vehicle brake initial velocity, $J_{\omega i \max }$ - the maximum moment of inertia, and $\eta$ - adjustment factor).

\section{Simulation analysis}

The main parameters used in the co-simulation are shown in Table 1, and it is assumed that the vehicle will brake in a straight line on a dry asphalt road at a constant speed.

Table 1 Main simulation parameters

\begin{tabular}{|l|c|c|}
\hline Quantity & Symbol & Value \\
\hline Half vehicle mass & $m$ & $800 \mathrm{~kg}$ \\
\hline Wheel inertia & $J$ & $0.5\left(\mathrm{~kg} \cdot \mathrm{m}^{2}\right)$ \\
\hline Acceleration of gravity & $g$ & $9.8 \mathrm{~m} / \mathrm{s}^{2}$ \\
\hline Wheel radius & $R$ & $0.36 \mathrm{~m}$ \\
\hline Braking initial speed & $v_{0}$ & $80 \mathrm{~km} / \mathrm{h}$ \\
\hline ABS failure speed & $v_{1}$ & $10 \mathrm{~km} / \mathrm{h}$ \\
\hline
\end{tabular}

According to the simulation results, the control algorithm was constantly improved. The simulation results were compared to the results the classic PID control algorithm. The results verify the proposed algorithm based on slip ratio control, which includes the sliding mode control and classic PID control, and it can be concluded that the sliding mode control is more suitable than that of other algorithms in a vehicle equipped with an EMB system. The cosimulation was carried out based on the simulation model and control model, as shown in Figure 4.

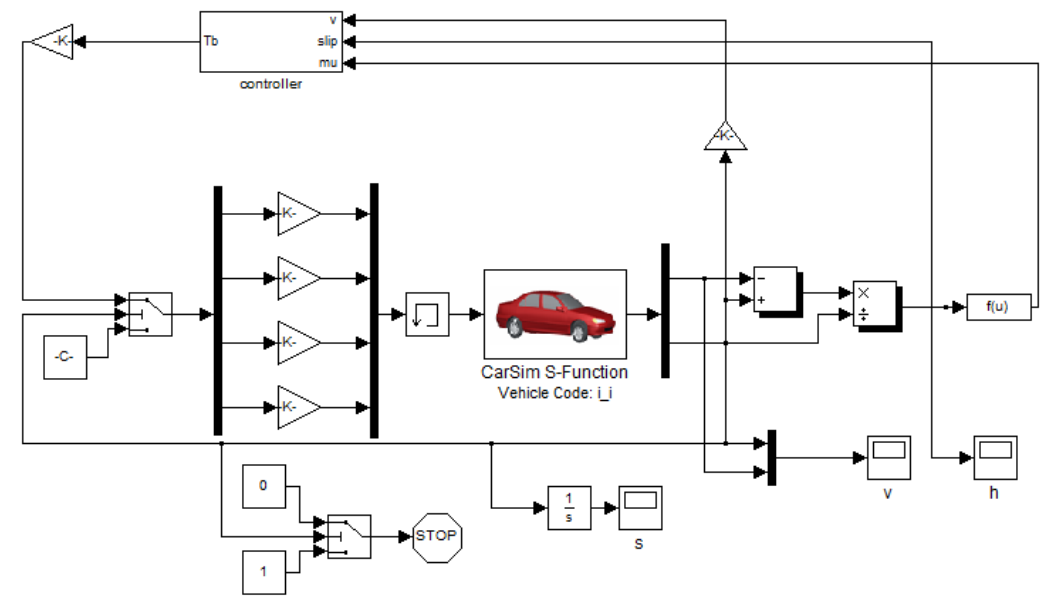

Fig. 4 Simulation model of ABS based on the Slip Ratio Control 
Figure 5 and Figure 6 show the vehicle and wheel speeds based on an electromechanical brake device controlled by the sliding mode and the classic PID. It is observed from Figure 5 and Figure 6 that the two algorithms, based on slip ratio control, can achieve a good anti-lock brake function performance. The stopping time with sliding mode control is slightly shorter than that of the classic PID control. It is observed from Figure 6 that large fluctuations in wheel speed exist at the beginning of braking, which has a greater impact on the braking performance.

Figure 7 and Figure 8 present the slip ratios of the four wheels controlled by the sliding mode control and classic PID control, respectively. The comparison shows that the wheels controlled by the two algorithms both can track over the desired slip rate well on dry asphalt.

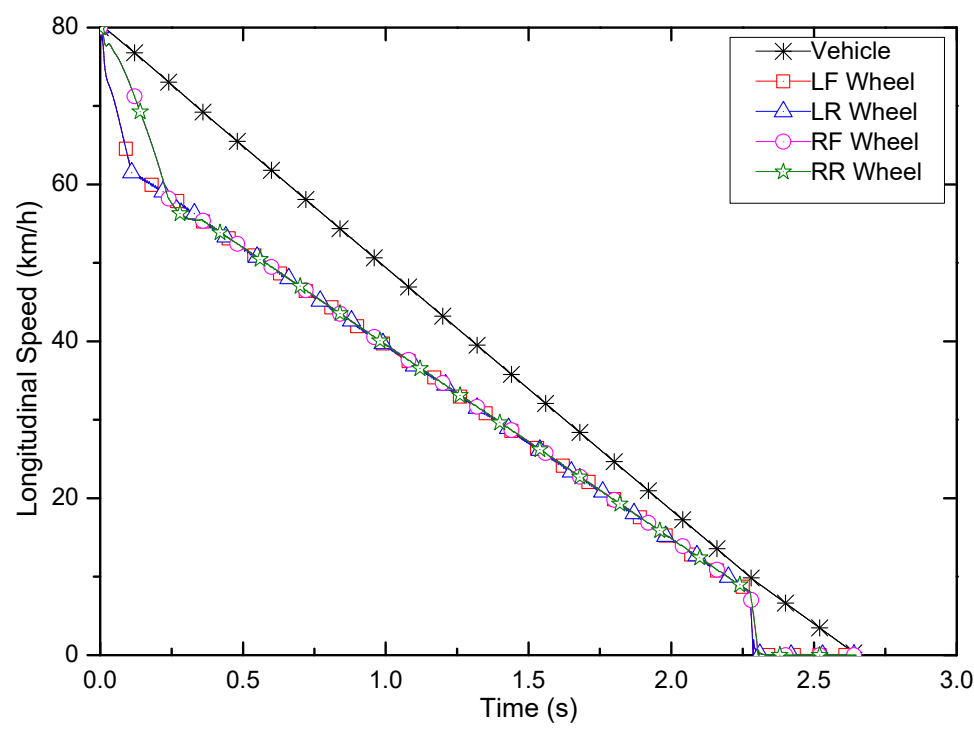

Fig. 5 Speeds of the vehicle and wheels based on SMC

Therefore, the vehicle with the electromechanical brake device installed can not only make better use of the longitudinal adhesion coefficient and shorten the brake distance but it can also obtain a larger lateral adhesion coefficient, which improves handling and stability during emergency braking. However, as observed from Figure 8, the vehicle cannot track over the desired slip rate exactly before $1 \mathrm{~s}$. At the same time, in the simulation process, it is found that the classic PID control parameters needs to be tuned, and it is easy for the system to become unstable due to its high sensitivity. 


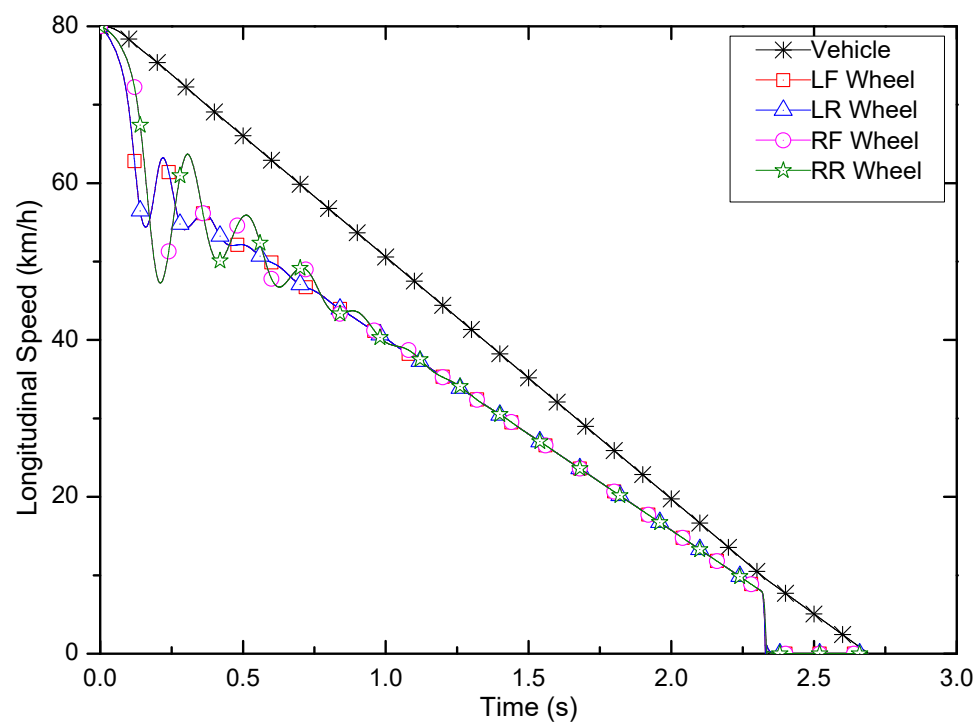

Fig. 6 Vehicle and wheel speeds based on classic PID

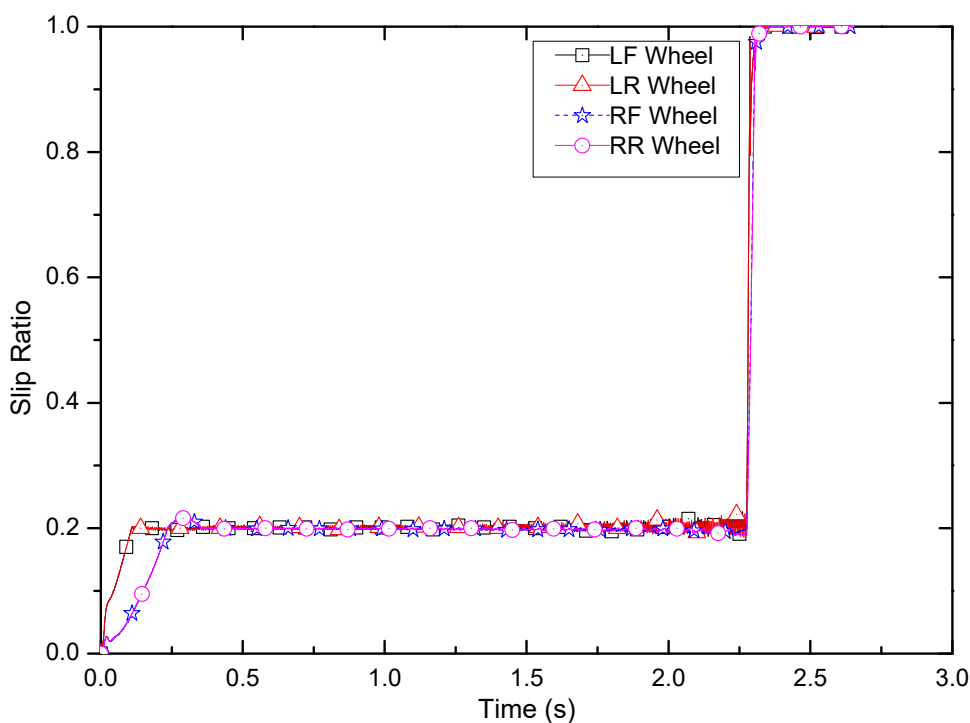

Fig. 7 Slip ratios of four wheels based on SMC

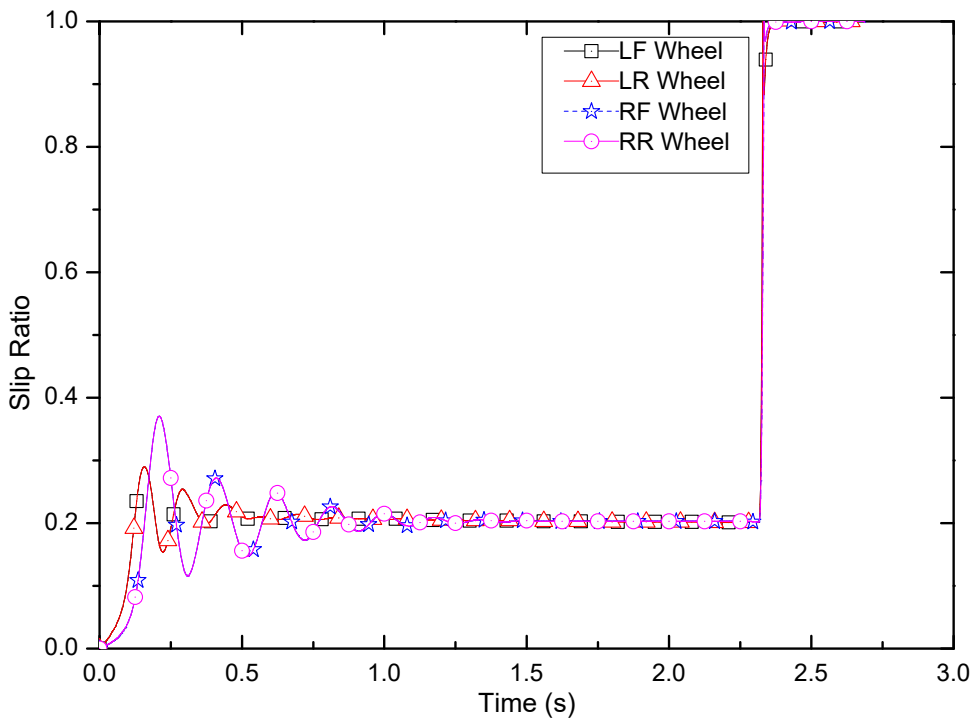

Fig. 8 Slip ratios of four wheels based on classic PID 


\section{Conclusions}

In this paper, braking dynamics were analyzed based on a two-wheel vehicle model. Then, an electromechanical brake device was designed and a simulation model of a vehicle ABS based on the electromechanical brake device was established. The anti-lock brake system was regulated using the slip ratio control method, as well as sliding mode control and classic PID control.

A co-simulation was carried out to verify the effectiveness of the application of sliding mode control and classic PID control on the electromechanical brake device.

\section{Acknowledgements}

This work is partially supported by the China Postdoctoral Science Foundation (Grant No. 2016M601320).

\section{REFERENCES}

[1] S Delprat. High order sliding mode control for hybrid vehicle stability, International Journal of Systems Science 2014, 45(5), 1202-1212. https://doi.org/10.1080/00207721.2012.745241

[2] V I Utkin; H C Chang. Sliding mode control on electro-mechanical systems, Mathematical problems in Engineering 2002, 8(4-5), 451-473. https://doi.org/10.1080/10241230306724

[3] C. Unsal; P Kachroo. Sliding mode measurement feedback control for antilock braking systems, Control Systems Technology 1999, IEEE Transactions on, 7(2), 271-281. https://doi.org/10.1109/87.748153

[4] R. Bhandari; S. Patil. Surface prediction and control algorithms for anti-lock brake system, Transportation research part C: emerging technologies 2012, 21(1), 181-195. https://doi.org/10.1016/j.trc.2011.09.004

[5] C. F. Hsu; T. C. Kuo. Adaptive exponential-reaching sliding-mode control for antilock braking systems, Nonlinear Dynamics 2014, 77(3), 993-1010. https://doi.org/10.1007/s11071-014-1357-6

[6] T. Kawabe; M. Nakazawa; I. Notsu; Y. Watanabe. A sliding mode controller for wheel slip ratio control system, Vehicle System Dynamics 1997, 27(5-6), 393-408. https://doi.org/10.1080/00423119708969338

[7] B. Ganji; A. Z. Kouzani; S.Y. Khoo; M. Shams-Zahraei. Adaptive cruise control of a HEV using sliding mode control, Expert Systems with Applications 2014, 41(2), 607-615. https://doi.org/10.1016/j.eswa.2013.07.085

[8] R. De Castro; R. E. Araújo; D. Freitas. Wheel slip control of EVs based on sliding mode technique with conditional integrators, Industrial Electronics 2013, IEEE Transactions on, 60(8): 3256-3271. https://doi.org/10.1109/tie.2012.2202357

[9] Y. Lin; C. Shen, J. Wang. A Simulation Study on Fuzzy Control of Anti-Lock Characteristic of Brake-byWire System, Journal of Highway and Transportation Research and Development 2006, 23(10), 124-127.

[10] E. J. Park; D. Stoikov. A Performance Evaluation of an Automotive Magnetorheological Brake Design with a Sliding Mode Controller, Mechatronics 2006, 16(7), 405-416. https://doi.org/10.1016/j.mechatronics.2006.03.004

[11] X. Y. Peng; C. R. Chen. Study of the Sliding Mode Control of Electromechanical Brake Systems, Journal of Hunan University: Natural Sciences 2010, 37(8), 35-39.

[12] J. Y. Wong. Theory of Ground Vehicles, John Wiley \& Sons 2008, New York.

[13] T. D. Gillespie. Fundamentals of Vehicle Dynamics, Society of Automotive Engineers Inc 1992, Warrendale.

[14] Gao, W.; Farahani, M. R.; Aslam, A.; Hosamani, S. Distance learning techniques for ontology similarity measuring and ontology mapping, CLUSTER COMPUT 20 2017, 959-968. https://doi.org/10.1007/s10586-017-0887-3

[15] Sabir, M.M., Electrohydrodynamic flow solution in ion drag in a circular cylindrical conduit using hybrid neural network and genetic algorithm. Kuwait Journal of Science, 2018. 45(1),20-28.

[16] Li, Y., The present situation and countermeasure analysis of Chinese biological medicine industry development. Journal of Mechanical Engineering Research and Developments, 2016. 39(4), 1000-1004. 
[17] Sahin, R. and L. Peide, Some approaches to multi criteria decision making based on exponential operations of simplified neutrosophic numbers. Journal of Intelligent and Fuzzy Systems: Applications in Engineering and Technology, 2017. 32(3), 2083-99. https://doi.org/10.3233/jifs-161695

[18] E. Bakker; H. B. Pacejka. A New Tire Model with an Application in Vehicle Dynamics Studies, $S A E$ Technical Paper 890087 1989. https://doi.org/10.4271/890087

[19] S. W. Zhou; Q. M. Chen. A Kind of Electromechanical Braking Device, China Patent 201410664221.9 2015.

[20] J. Guo; X. Jian; G. Lin. Performance Evaluation of an Anti-Lock Braking System for Electric Vehicles with a Fuzzy Sliding Mode Controller, Energies 2014, 7(10), 6459-6476. https://doi.org/10.3390/en7106459

[21] C. Shen; J. Wang; Y. Lin. Study on Brake Actuator of Electromechanical Braking System, Transactions of the Chinese Society for Agricultural Machinery 2007, 38(8), 30-33.

[22] Gao, W.; Wang, W. The fifth geometric-arithmetic index of bridge graph and carbon nanocones, $J$ DIFFER EQU APPL 23 2017, 100-109. https://doi.org/10.1080/10236198.2016.1197214

Submitted: $\quad$ 20.7.2017

Accepted: $\quad$ 27.3.2019
Shuwen Zhou $\bowtie$

Qingming Chen

School of Mechanical Engineering and

Automation, Northeastern University, Shenyang 110819, China.

Siqi Zhang

School of Transportation Engineering, Shenyang Jianzhu University, Shenyang 110168, China

Corresponding Author e-mail shwzhou@mail.neu.edu.cn 